\title{
A novel mutation in ITGB4 gene in a newborn with epidermolysis bullosa, pyloric atresia, and aplasia cutis congenita
}

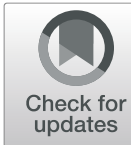

Emel Okulu*, Ceren D. Durmaz², Gaffari Tunc ${ }^{1}$, Adil Guzel³ ${ }^{3}$ Nuket Y. Kutlay², Omer Erdeve ${ }^{1}$, Begum Atasay ${ }^{1}$, John A. McGrath", Lu Liư and Saadet Arsan ${ }^{1}$

\begin{abstract}
Background: Epidermolysis bullosa with pyloric atresia (EB-PA), also known as Carmi syndrome, is an uncommon, autosomal recessive genodermatosis that typically affects the skin and gastrointestinal tract. EB-PA is caused by homozygous or compound heterozygous mutations in the integrin alpha 6 (ITGA6) gene on chromosome 2q31.1 or in the integrin beta 4 (ITGB4) gene on 17q25.1.

Case presentation: A male premature infant was born with aplasia cutis, atresia of the pylorus, and bilateral hydronephrosis. His clinical and imaging findings were compatible with EB-PA. A novel, small deletion of the last two bases in exon 6 and the first two nucleotides of intron 6 (c.565_566+2del) in ITGB4 gene was identified.

Conclusion: EB-PA-aplasia cutis congenita is known to be a non-treatable condition with a poor prognosis as the reported case. The novel mutation reported in this patient may lead to the lethal form of this disease. Identification of underlying genetic abnormality is critical to give genetic counseling.
\end{abstract}

Keywords: Epidermolysis bullosa, Carmi syndrome, Integrin alpha 6, Integrin beta 4

\section{Background}

Epidermolysis bullosa (EB) is a clinically and genetically heterogeneous group of inherited skin diseases characterized by blisters followed by skin and mucosal erosions. EB has subgroups based on the ultrastructural level. There are four subgroups: EB simplex, junctional epidermolysis bullosa (JEB), dystrophic EB, and Kindler syndrome [1-3].

JEB with pyloric atresia (JEB-PA, OMIM: 226730) is a rare form of EB with multisystem involvement. Cutaneous manifestations, congenital pyloric atresia, and ureteral and renal anomalies such as renal collecting system defects, multicystic/dysplastic kidney, hydronephrosis, and absent bladder are the characteristic features of the disorder [4]. Cutaneous manifestations of

\footnotetext{
* Correspondence: emelokulu@gmail.com

'Division of Neonatology, Department of Pediatrics, Ankara University Faculty of Medicine, Tip Fakultesi Street, 06590 Mamak, Ankara, Turkey

Full list of author information is available at the end of the article
}

JEB-PA include severe mucocutaneus blisters, extreme skin fragility, atrophic scarring, and milia (small white spots). JEB-PA is caused by mutations in the ITGA6 or ITGB4 genes coding for subunit alpha $6(\alpha 6)$ or beta 4 $(\beta 4)$ of integrin $[5,6]$. Additional features shared by JEBPA include aplasia cutis congenita (ACC) that means congenital localized absence of the skin affecting the extremities, head, nail dystrophy, scarring alopecia, enamel hypoplasia, contractures, and dilated cardiomyopathy [7-9].

ITGA6, mapped to $2 \mathrm{q} 31.1$, is one of the genes known to cause this phenotype. ITGA6 consists of 28 exons and encodes the integrin $\alpha 6$ which is a member of integrin alpha chain family. On the other hand, ITGB4 is located at $17 \mathrm{q} 25.1$ and encodes integrin $\beta 4$ which is a component $\alpha 6 \beta 4$ integrin with integrin $\alpha 6$. Most of the patients with JEB-PA bear pathogenic variants in ITGB4 gene, while ITGA6 mutations are less common. Integrins are heterodimeric transmembrane receptors that play a 
critical role in cell surface adhesion and signaling. The a6ß4 integrin is a hemidesmosomal protein, and its expression is altered by the mutations in ITGA6 and ITGB4 genes that cause structural defects in hemidesmosome [10-12].

Here, we describe a clinical case of a newborn with JEB-PA and ACC due to a novel mutation in ITGB4 gene.

\section{Case presentation}

A male preterm infant was born at 31 weeks' gestation by vaginal delivery as the first child to consanguineous parents with a birth weight of $1620 \mathrm{~g}$. On prenatal ultrasonography, gastric dilatation and unilateral hydronephrosis were described. He received positive pressure ventilation at the delivery room and transferred to neonatal intensive care unit after stabilization. On physical examination, the skin was absent on the face, anterior side of the left forearm, both legs, and scrotum. He had multiple bullous lesions on the right arm, along with dystrophic nails. The radiograph of the abdomen showed gastric bubble resembling that of pyloric stenosis (Figs. 1, 2 , and 3). Bilateral hydronephrosis and left ureterocele were detected by abdominal ultrasonography. Echocardiography and cranial ultrasonography were normal. The patient was intubated due to respiratory failure at the 2nd day of his life. Skin biopsy was performed and confirmed the diagnosis of EB. During follow-up, intravenous hydration was increased and parenteral nutrition and intravenous albumin were administered. He could

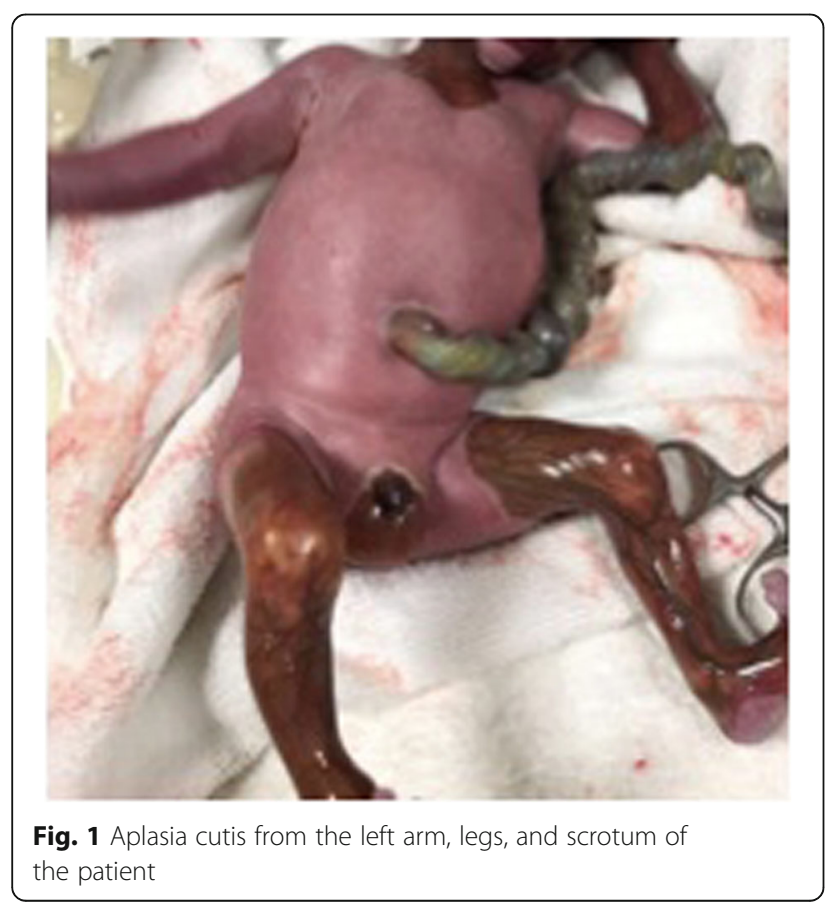

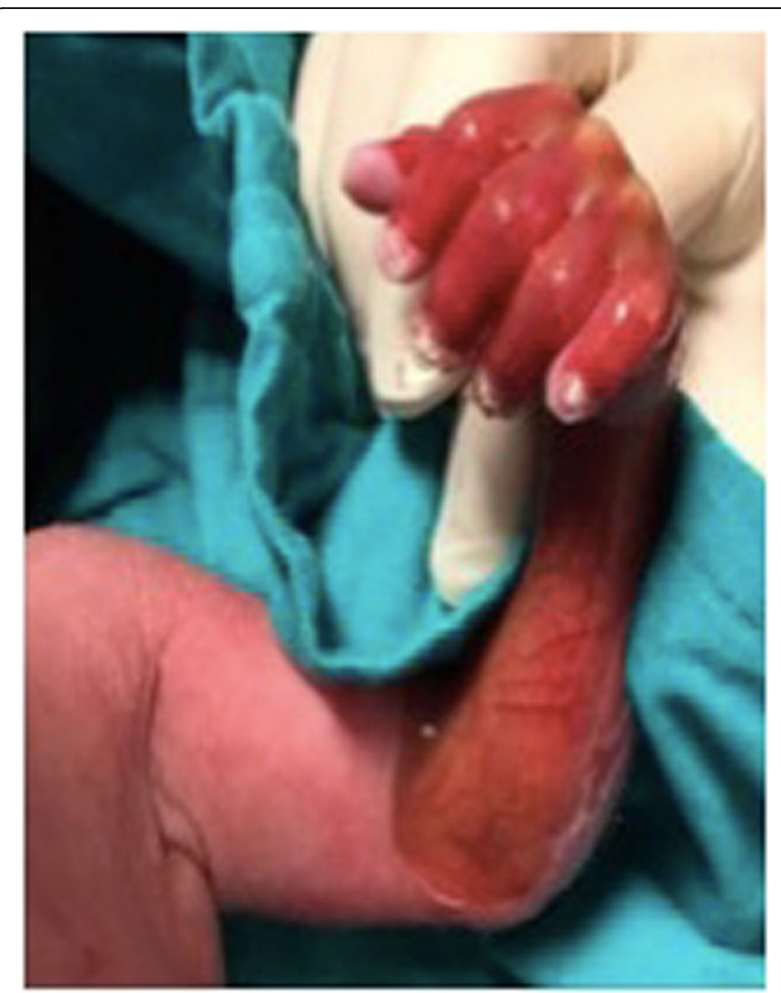

Fig. 2 Dystrophic nails of the patient

not achieve the required stability for surgery and died on the 4th day on his life.

Written informed consent was obtained from the parent of the patient.

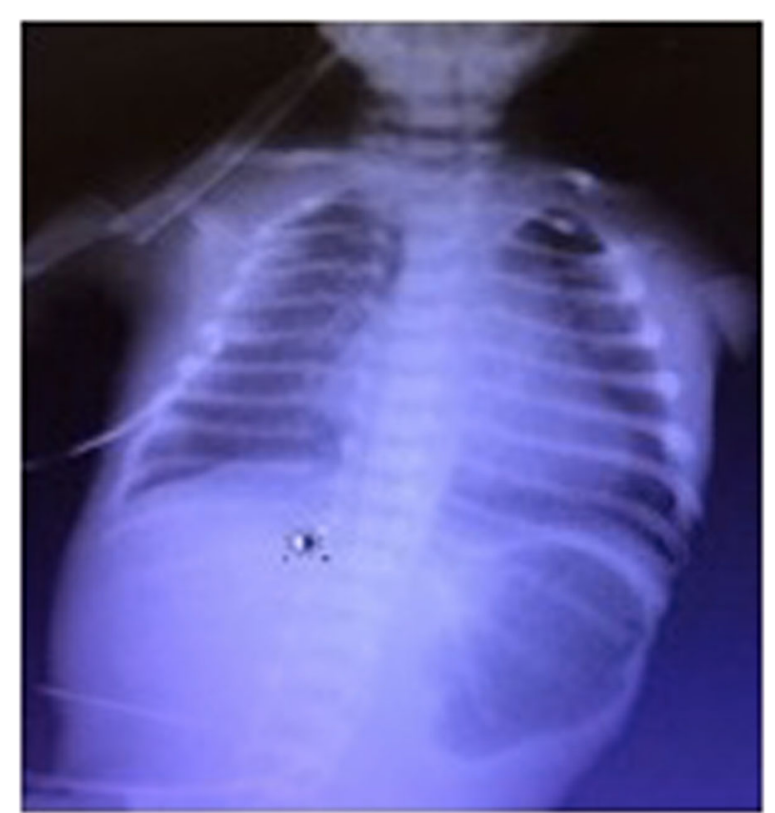

Fig. 3 Radiograph of the abdomen showing gastric dilatation due to pyloric atresia 


\section{Genetic studies}

Genomic DNA was isolated from $200 \mu \mathrm{L}$ of peripheral blood sample of the patient and his parents using Magna Pure LC DNA Isolation Kit-Large Volume and Magna Pure LC instrument (Roche Applied Science, Mannheim, Germany). All exons and the flanking regions of ITGA6 and ITGB4 were amplified by polymerase chain reaction using the $100 \mathrm{ng}$ genomic DNA (the 260/280 ratios of DNA samples were $\sim 1.8$ ) as a template for each PCR reaction. Conditions and primers for generating PCR products spanning all exons of the coding regions and flanking intronic sequences of the genes have been previously described elsewhere [6, 13]. After that, bidirectional Sanger sequencing analysis was performed using BigDye Terminator v3.1 Cycle Sequencing Kit on an ABI PRISM 3130 Genetic Analyzer (Thermo Fisher Scientific, Waltham, MA, USA).

A novel homozygous c.565_566+2del mutation was found in ITGB4 gene of the patient. His parents were found to be heterozygous for the same mutation (Fig. 4). This homozygous deletion in ITGB4 disrupts the intron 6 donor splice site and expected to result in aberrant splicing and loss of function for the encoded $\beta 4$ integrin subunit. This variant was not listed in the $1000 \mathrm{Ge}$ nomes (http://browser.1000genomes.org) or in the ExAC database (http://exac.broadinstitute.org).

\section{Discussion}

Here, we describe a case with JEB-PA and ACC emerging from a novel splice site mutation in ITGB4 gene. $\mathrm{EB}$ is an inherited disease with an estimated frequency at 1 in 300,000, whereas pyloric atresia (PA) has an incidence of 1 in 100,000 live births. Familial PA with EB was first described in 1968, and pathophysiology was first described by Carmi, so the disease has been called as "Carmi syndrome." Gastrointestinal, urinary, pulmonary, and eye involvement are also reported to be associated with EB-PA. ACC has been previously described with EB-PA in few reports. Literature shows that JEBPA-ACC is the most severe spectrum of the disease and suggested that ACC is the poorest prognostic factor of the combination $[7-9,11,14]$.

Mutations in ITGA6 and ITGB4 cause an altered expression of $\alpha 6 \beta 4$ integrin resulting in a structural defect of hemidesmosome which has an important role in stabilizing the association of the dermis with the epidermis. The defective hemidesmosomes cause blisters and erosions on the skin and gastrointestinal and urogenital abnormalities $[11,15]$. Mutations in ITGA6 are less frequent than in ITGB4 [16]. There are more than $80 \mathrm{mu}-$ tations that cause EB-PA and some of them are lethal. In lethal cases, the expression of $\alpha 6 \beta 4$ integrin is absent [17]. We identified a novel, small deletion of the last two bases in exon 6 and first two nucleotides of intron 6 (c.565_566+2del) in ITGB4 gene in our patient with Carmi syndrome and ACC. This mutation causes splicing defect and premature stop codon which is responsible for the clinical features of our patient.

\section{Conclusions}

EB-PA-ACC is known to be a non-treatable condition with a poor prognosis. There are few patients reported to be survived [16]. Although our patient was a premature baby and intestinal surgery could not be performed, we thought that the novel mutation reported in this patient may lead to a lethal form of the disease. Identification of underlying genetic abnormality is critical to give genetic counseling.

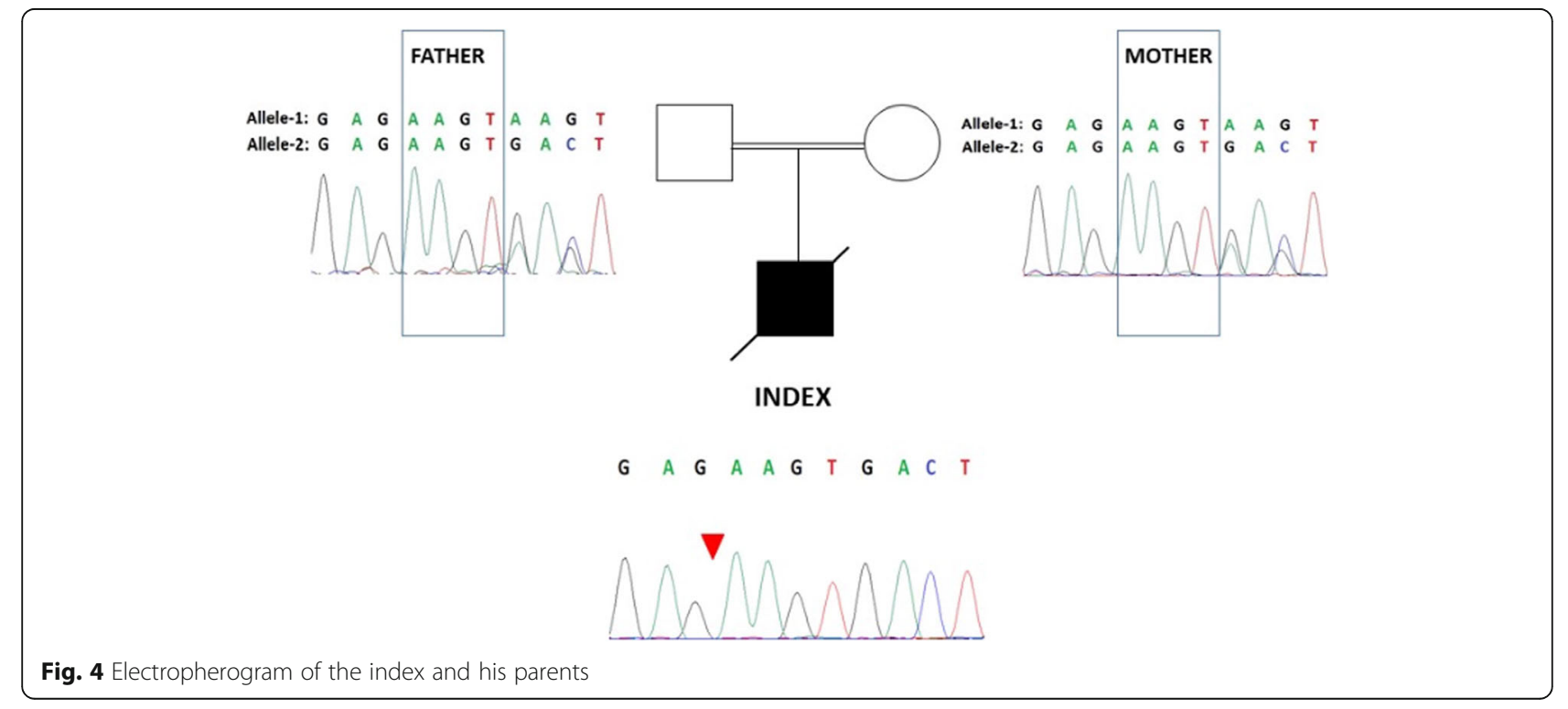




\section{Abbreviations}

ACC: Aplasia cutis congenita; EB: Epidermolysis bullosa; EB-PA: Epidermolysis bullosa with pyloric atresia; JEB: Junctional epidermolysis bullosa; JEBPA: Junctional epidermolysis bullosa with pyloric atresia; JEB-PA-

ACC: Junctional epidermolysis bullosa with pyloric atresia and aplasia cutis congenita

\section{Acknowledgements}

None.

\section{Authors' contributions}

GT and EO contributed to the conception. CDD, AG, EO, NYK, McGJ, and LL contributed to the acquisition and analysis. NYK, OE, McGJ, and LL contributed to the interpretation of the data. GT, CDD, and EO drafted the work. NYK, OE, BA, and SA critically revised the manuscript. GT, CDD, AG, EO, NYK, OE, BA, SA, McGJ, and LL approved the final manuscript.

\section{Funding}

None.

\section{Availability of data and materials}

Not applicable.

\section{Ethics approval and consent to participate}

Not applicable.

\section{Consent for publication}

Written parental informed consent was obtained for the publication of this clinical report and images.

\section{Competing interests}

The authors declare that they have no competing interests.

\section{Author details}

'Division of Neonatology, Department of Pediatrics, Ankara University Faculty of Medicine, Tip Fakultesi Street, 06590 Mamak, Ankara, Turkey. ²Department of Medical Genetics, Ankara University Faculty of Medicine, Ankara, Turkey. ${ }^{3}$ Department of Pediatrics, Ankara University Faculty of Medicine, Ankara, Turkey. ${ }^{4}$ St John's Institute of Dermatology, King's College London (Guy's Campus), London, UK. ${ }^{5}$ Viapath, St. Thomas' Hospital, London, UK.

Received: 10 October 2019 Accepted: 28 February 2020

Published online: 01 April 2020

\section{References}

1. Fine JD, Eady RA, Bauer EA, Bauer JW, Bruckner-Tuderman L, Heagerty A et al (2008) The classification of inherited epidermolysis bullosa (EB): report of the Third International Consensus Meeting on Diagnosis and Classification of EB. J Am Acad Dermatol 58:931-950

2. Fine JD (2010) Inherited epidermolysis bullosa: recent basic and clinical advances. Curr Opin Pediatr 22:453-458

3. Fine JD, Bruckner-Tuderman L, Eady RA, Bauer EA, Bauer JW, Has C, Heagerty A et al (2014) Inherited epidermolysis bullosa: updated recommendations on diagnosis and classification. J Am Acad Dermatol 70: 1103-1126

4. Pfendner EG, Lucky AW (1993) Epidermolysis bullosa with pyloric atresia. In: Adam MP, Ardinger HH, Pagon RA, Wallace SE, LJH B, Stephens K, Amemiya A (eds). GeneReviews (R), Seattle

5. Vidal F, Aberdam D, Miquel C, Christiano AM, Pulkkinen L, Uitto J et al (1995) Integrin beta 4 mutations associated with junctional epidermolysis bullosa with pyloric atresia. Nat Genet 10:229-234

6. Pulkkinen L, Kimonis VE, XU Y, Spanou EN, McLean WH, Uitto J (1997) Homozygous alpha6 integrin mutation in junctional epidermolysis bullosa with congenital duodenal atresia. Hum Mol Genet 6:669-674

7. Maman E, Maor E, Kachko L, Carmi R (1998) Epidermolysis bullosa, pyloric atresia, aplasia cutis congenita: histopathological delineation of an autosomal recessive disease. Am J Med Genet 78:127-133

8. Birnbaum RY, Landau D, Elbedour K, Ofir R, Birk OS, Carmi R (2008) Deletion of the first pair of fibronectin type III repeats of the integrin beta-4 gene is associated with epidermolysis bullosa, pyloric atresia and aplasia cutis congenita in the original Carmi syndrome patients. Am J Med Genet A 146A:1063-1066

9. Kayki G, Bozkaya D, Ozaltin F, Orhan D, Kaymaz F, Kaymaz E et al (2017) Epidermolysis bullosa with pyloric atresia and aplasia cutis in a newborn due to homozygous mutation in ITGB4. Fetal Pediatr Pathol 36:332-339

10. Charlesworth A, Gagnoux-Palacios L, Bonduelle M, Ortonne JP, De Raeve L, Meneguzzi G (2003) Identification of a lethal form of epidermolysis bullosa simplex associated with a homozygous genetic mutation in plectin. J Invest Dermatol 121:1344-1348

11. Chung HJ, Uitto J (2010) Epidermolysis bullosa with pyloric atresia. Dermatol Clin 28:43-54

12. Kim JH, Park HY, Lee HJ, Eom M, Choi EH (2011) Case of epidermolysis bullosa with pyloric atresia. Ann Dermatol 23 Suppl:S41-S44

13. Pulkkinen L, Kim DU, Uitto J (1998) Epidermolysis bullosa with pyloric atresia: novel mutations in the $\beta 4$ integrin gene (ITGB4). Am J Pathol 152: 157-166

14. Pulkkinen L, Uitto J (1999) Mutation analysis and molecular genetics of epidermolysis bullosa. Matrix Biol 18:29-42

15. Geuijen CA, Sonnenberg A (2002) Dynamics of the alpha6beta4 integrin in keratinocytes. Mol Biol Cell 13:3845-3858

16. Nakano A, Pulkkinen L, Murrell D, Rico J, Lucky AW, Garzon M et al (2001) Epidermolysis bullosa with congenital pyloric atresia: novel mutations in the beta 4 integrin gene (ITGB4) and genotype/phenotype correlations. Pediatr Res 49:618-626

17. Pulkkinen L, Rouan F, Bruckner-Tuderman L, Wallerstein R, Garzon M, Brown T et al (1998) Novel ITGB4 mutations in lethal and nonlethal variants of epidermolysis bullosa with pyloric atresia: missense versus nonsense. Am J Hum Genet 63:1376-1387

\section{Publisher's Note}

Springer Nature remains neutral with regard to jurisdictional claims in published maps and institutional affiliations.

\section{Submit your manuscript to a SpringerOpen ${ }^{\circ}$ journal and benefit from:}

- Convenient online submission

- Rigorous peer review

- Open access: articles freely available online

- High visibility within the field

- Retaining the copyright to your article

Submit your next manuscript at $\boldsymbol{\nabla}$ springeropen.com 